\title{
Remarkable extension of PAI-1 half-life surprisingly brings no changes to its structure
}

\author{
JERZY JANKUN ${ }^{1,3,4}$, JIE YANG $^{2}$, HONG ZHENG $^{2}$, FRANK Q. HAN ${ }^{2}$, \\ ABDULRAHMAN AL-SENAIDY ${ }^{4}$ and EWA SKRZYPCZAK-JANKUN ${ }^{1}$ \\ ${ }^{1}$ Urology Research Center, Department of Urology, The University of Toledo, Health Science Campus, Toledo, \\ OH 43614; ${ }^{2}$ Structure Based Design, Inc., San Diego, CA 92121, USA; ${ }^{3}$ Department of Clinical Nutrition, \\ Medical University of Gdańsk, 80-211 Gdańsk, Poland; ${ }^{4}$ Protein Research Chair, Department \\ of Biochemistry, College of Sciences, King Saud University, Riyadh, Saudi Arabia
}

Received August 1, 2011; Accepted September 5, 2011

DOI: $10.3892 /$ ijmm.2011.798

\begin{abstract}
Plasminogen activator inhibitor type 1 (PAI-1) is a serpin protein, a natural inhibitor of urokinase (uPA) and tissue plasminogen activators (tPA). By inhibiting uPA it can block growth of the cancer tumors by suppressing angiogenesis, while when acting on tPA in the blood it can avert conversion of plasminogen to plasmin preventing lysis of the clot. Furthermore, blocking PAI-1 activity can protect against thrombosis. Thus PAI-1 makes great impact on human homeostasis and is desirable for clinical application. Wild-type PAI-1 (wt-PAI-1) has a short span of activity with a $\mathrm{t}_{1 / 2}$ of $\sim 2 \mathrm{~h}$, being spontaneously converted into a latent form. An enormous effort has been made to create a more stable molecule with $>600$ PAI-1 variants constructed to study its structure-function relationship. In the present study, we evaluate the structure of the active recombinant VLHL-PAI-1 (very long half life, active $>700 \mathrm{~h}$ ) which is glycosylated similarly to wt-PAI-1 at N232 and N288, with the extended reactive center loop, intact engineered -S-S-bridge (Q174C, $\mathrm{G} 323 \mathrm{C}$ ) that precludes latency without affecting structure, and can be controlled by a reducing agent to terminate activity at will. We have already proven its usefulness to control cancer in human cancer cells, as well as preventing clot lysis in human whole blood and plasma and in a mouse model. Our results demonstrate the potential therapeutic applications (topical or systemic) of this protein in the treatment of cancer, for the trauma patients to ward off an excessive blood loss, or for people with the PAI-1 deficiency, especially during surgery.
\end{abstract}

Correspondence to: Dr Jerzy Jankun, Urology Research Center, Department of Urology, The University of Toledo, Health Science Campus, 3000 Arlington Ave., Toledo, OH 43614, USA

E-mail: jerzy.jankun@utoledo.edu

Key words: plasminogen activator inhibitor type 1, therapy, disulfide bridge

\section{Introduction}

Proteins naturally produced in the body could be of great clinical value as non-immunogenic agents, if their function can be protected for a therapeutically significant period of time. Engineering proteins by single-point or multiple-point mutations always raise question how far the expressed mutant deviates from the wild type (wt) protein, especially if some dramatic changes in protein characteristics are observed. Maintaining $100 \%$ of VLHL PAI-1 starting activity for about 700 times longer than wt-PAI-1 in the parallel experiments at the same conditions $(50 \mathrm{mM}$ Tris $\mathrm{HCl}, 150 \mathrm{mM} \mathrm{NaCl}$, $\mathrm{pH} 7.5$, incubated with $100 \mu \mathrm{g} / \mathrm{ml}$ of bovine serum albumin at room temperature) is such a dramatic change (1). The X-ray analysis is the only method for larger proteins to provide solid evidence about similarities and structural properties, but it requires orderly crystals to produce the desired diffraction data. For such crystals to form, the protein solution has to be chemically and structurally homogeneous over an extended period of time. Crystallizing wt-PAI-1 ( $\left.\mathrm{t}_{1 / 2} \sim 2 \mathrm{~h}\right)$ was a bewildering challenge since its active form with a flexible, far out extended reactive center loop (RCL, encompassing |355-373|, also denoted as P14-P4'), converts within hours into radically different, latent (inactive) form where this loop slides inside a $\beta$ sheet as its central strand (A4). This changes the size and shape of the entire molecule causing structural heterogeneity. Thus, only the structure determination of the latent form of the recombinant wt-PAI-1 enzyme was possible [PDB ID: 1c5g, 1dvn (2,3) and 1lj5 (Stein and Baek, revised in 2009)]. However, experiments with functionally extended mutants $\left(\mathrm{t}_{1 / 2}\right.$ ranging from several to $\sim 180 \mathrm{~h}$ ) were more successful. To avoid confusion and assure consistency when discussing mutations we use human PAI-1 sequence numbering after Swiss-Prot P05121 chain 24-402 with P1-P1' at R369-M370, regardless of the numbers used in the original publication. The known structures describe PAI-1 so called 14-1B, containing four mutations N173H, K177T, Q342L, M377I [PDB ID: 1b3k, 1dvm, loc0 (missing |361-370I)] (2,4), and another with additional point mutation at Q324P (PDB ID: 1db2) (5). Another recent PDB entry 3pb1 describes the complex of the PAI-1 
14-1B mutant with the enzymatically inactive uPA S195A mutant, giving valuable information about the complex formation between these molecules (6). The most recently deposited structure PDB ID: 3q02 contains only one mutation (W198F) and has stability superior over these listed above $\left(\mathrm{t}_{1 / 2} \sim 400 \mathrm{~h}\right.$ and 18 times better than wt-PAI- 1 at the same conditions) (7) but like 1 loc 0 does not depict the reactive loop due to a disorder (missing |355-370I) (8). Natural PAI-1 is glycosylated at two asparagines: 232 and 288, but except for cleaved PAI-1 where it was confirmed in the crystal structure (PDB ID: $1 \mathrm{a} 7 \mathrm{c}$, with pentapeptides mimicking A4- $\beta$-strand thus having a molecule core as in the latent form) (9), this information is otherwise not provided.

In our efforts to create a stable and active PAI-1 we rationally designed to introduce a $-\mathrm{S}-\mathrm{S}$ bond by replacing Q197 and G355 with cysteines that would hold together A3 and A5 strands of the central $\beta$-sheet being the core of this molecule and thus preserving activity by preventing retraction of the RCL (Fig. 1). We have found that in the presence of the reducing agent the disulfide bridge breaks and the molecule converts to the latent form, but when this reducer is removed the molecule spontaneously rebuilds -S-S- and regains activity (10). This molecule shows no decline in activity for a month at room temperature (testing ceased after $700 \mathrm{~h}$ ) in contrast to other known functionally stabilized mutants for which $t_{1 / 2}$ does not exceed 16 days (7). Such long undeterred activity assures adequate stability in storage and treatment in the future possible clinical applications.

\section{Materials and methods}

VLHL PAI-1 expression and purification. Glycosylated VLHL-PAI-1 was expressed in insect cells (Sf9) and purified to $98 \%$ according to a previously described protocol (11). Briefly, cell lysate was applied to a $\mathrm{Ni}^{2+}-\mathrm{NTA}$ agarose column pre-equilibrated with a buffer $(20 \mathrm{mM}$ sodium acetate, $\mathrm{pH}$ 5.6, $0.5 \mathrm{M} \mathrm{NaCl}, 5 \mathrm{mM}$ imidazole, and $0.01 \%$ Tween 80). The column was washed with the same buffer, then with the wash buffer $(20 \mathrm{mM}$ sodium acetate, $0.5 \mathrm{M} \mathrm{NaCl}, 60 \mathrm{mM}$ imidazole, and $0.01 \%$ Tween $80, \mathrm{pH} 5.6$ ), and eluted with the buffer containing a 60-1000 mM imidazole gradient. Column fractions containing active PAI-1 were pooled and loaded onto a desalting column equilibrated with a buffer $(0.025 \mathrm{M}$ phosphate buffer, $0.6 \mathrm{M} \mathrm{NaCl}, 1 \mathrm{mM}$ EDTA, $\mathrm{pH}$ 6.9). The column was washed and PAI-1 was eluted, concentrated and stored at $-80^{\circ} \mathrm{C}$.

Crystallization, data collection and structure determination. VLHL-PAI-1 at a concentration of $5 \mathrm{mg} / \mathrm{ml}$ in $0.025 \mathrm{M}$ phosphate buffer, $0.6 \mathrm{M} \mathrm{NaCl}, 1 \mathrm{mM}$ EDTA, pH 6.9, was crystallized by vapor diffusion in hanging drops using 1:1 mixture with $0.025 \mathrm{M} \mathrm{NaCl}, 27 \%$ t-butanol in $0.05 \mathrm{M}$ Tris $\mathrm{HCl} \mathrm{pH} 8.5$ at $295 \mathrm{~K}$. Crystallographic data were collected at Berkeley National Laboratory beam line 5.0.3, at $100 \mathrm{~K}$, processed with HKL suite v.0.95 to a $2.7 \AA$ resolution (12), solved by molecular replacement (CCP4 suite of programs) and refined by Refmac 5.6 to $\mathrm{R}=20.9 \%, \mathrm{R}_{\text {free }}=28.2 \%$ using 13038 independent reflections and a molecular model of 3086 atoms (13). Modeling to the electron density maps was performed using Coot (14), the figures were prepared utilizing CHAIN v.8 and

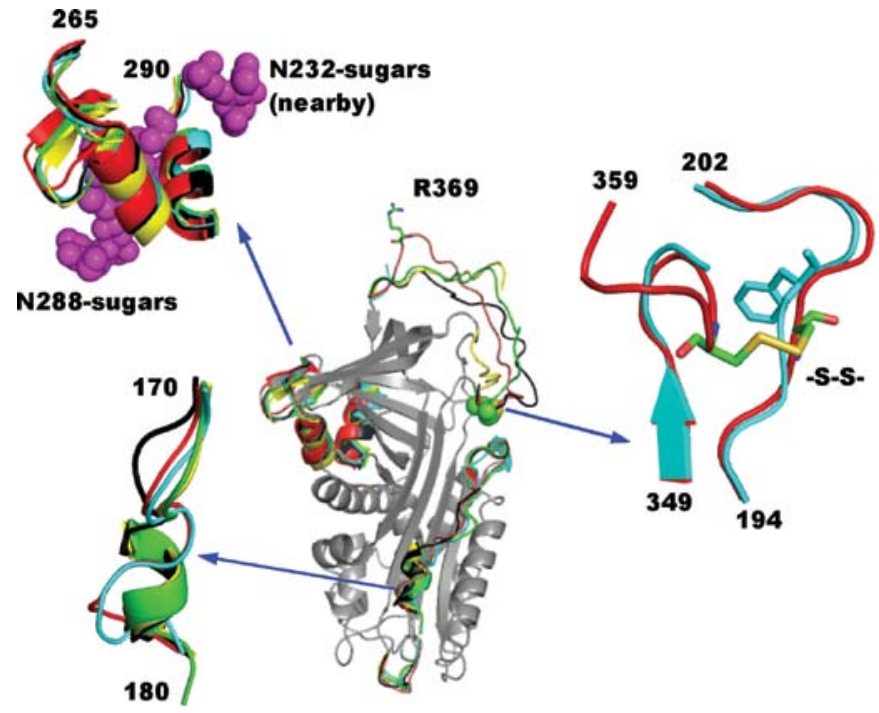

Figure 1. Superposition of PAI-1 structures in active form are shown in the center, the ribbon diagram with the secondary structure elements shown in grey where they coincide and in color for the disparate fragments. Red, 3r41; cyan, 3q02; green, 1b3k; yellow, 1dvm; black, 1db2; R369 shows position P1 and the spheres mark cysteines in 3r4l. Additional panels: at right, G355C and Q197C forming the disulfide bridge in VLHL-PAI-1 (3r41, red) and W198F in 3q02 (cyan); upper left, the glycosylation sites (3r41), asparagines and sugars shown as the space filling models in purple, N232 belongs to the nearby $\beta$-sheet exposed to solvent; the lower left panel illustrates the change to the helical structure in 14-1B mutants in contrast to the unstructured coil in the native sequence. The enlarged details are in arbitrary scale and orientation.

PyMOL $(15,16)$. The detailed relevant information, the sets of atomic coordinates and structure factors have been deposited in the Protein Data Bank entry 3r41.

\section{Results and Discussion}

Our X-ray analysis of VLHL-PAI-1 (deposited as PDB entry 3r4l) confirmed presence of the expanded RCL and a junction of 197-355 residues via -S-S- (Fig. 2a). Structural comparison with other active PAI-1 molecules (1b3k, 1dvm, $1 \mathrm{db} 2,3 \mathrm{q} 02$ and $3 \mathrm{r} 4 \mathrm{l}$ ) shows amazingly small structural differences with consensus scores rmsd of 0.53-1.11 $\AA$ and a Q-score of 0.77-0.83 [PDBeFold v. 2.36 (17)]. These PAI-1 mutants have $95-99 \%$ sequence identity and when aligned on the $\mathrm{C} \alpha$-backbone in a pairwise manner give an rmsd of $0.02 \AA$ ( $1 \mathrm{dvm}), 0.55 \AA$ (1db2), $0.28 \AA$ (3q02) and $0.50 \AA$ (3r4l) in relation to $1 \mathrm{~b} 3 \mathrm{k}$. While these overall values are negligible a graphical representation of the aligned structures clearly shows regions where they significantly differ (Fig. 1) indicating their high mobility, especially for the flexible coil of the RCL segment.

The impact of the mutations on the functional stability is obvious in the case of VLHL-PAI-1. Examination of the $197 \mathrm{C} \alpha \rightarrow 355 \mathrm{C} \alpha$ distance in the latent (PDB ID: 1c5g, 1dvn, 1lj5) and active forms (PDB ID: 1b3k, 1db2, 1dvm) of PAI-1 structures shows 4.1-4.2 $\AA$ in the latent and $3.4 \AA$, $4.4 \AA$ and $5.0 \AA$ in the active PAI-1 (respectively), which corresponds well to the $5.0 \AA$ $\mathrm{observed}$ in the structure with -S-S- reported in the present study (resolution $2.7 \AA$ ). Hence this distance is similar regardless of the PAI-1 form and comparable with 

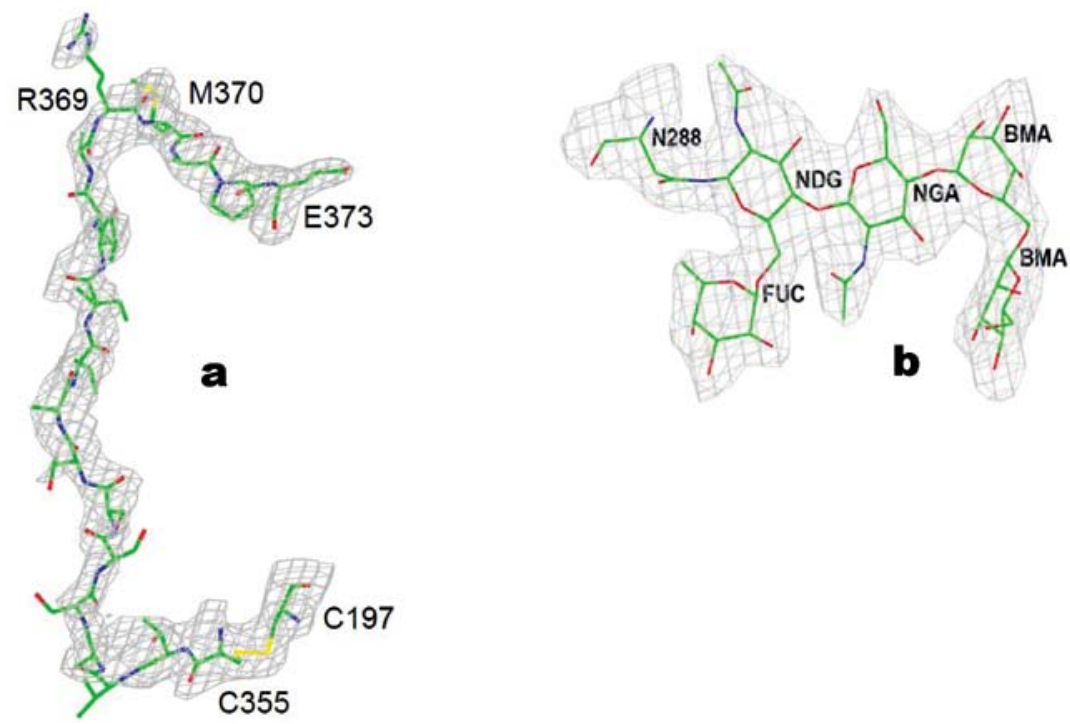

Figure 2. (a) Segment of the electron density map 2Fo-Fc contoured at 1o (grey) showing the reactive center loop P14-P4' and the disulfide bridge. (b) The same map near the N288 glycosylation site showing the identified sugars (a and b are in arbitrary scales).

the $\sim 5 \AA \mathrm{C} \alpha$...C $\alpha$ fusion observed between the A3...A5 $\uparrow \uparrow$ $\beta$-strands in this $\downarrow \uparrow \uparrow \downarrow \beta$-sheet of the active form. While it practically does not impact the overall trace of main chain (rmsd), the secondary or tertiary structure (of the active form), this -S-S- harness at the hinge region firmly holds these segments of molecule at $5 \AA$ distance thus precluding their rearrangement from the active to latent (inactive) form $\downarrow \uparrow \downarrow \uparrow \downarrow$ which requires partition of $\uparrow \uparrow$ by $>9 \AA$ for such insertion to occur. T357 should be placed between E350 and F194 which are the residues initiating the central hydrogen bonds zipper between the $\downarrow \uparrow \uparrow \downarrow$ parallel $\beta$-strands.

Our other mutant with the disulfide bridge joining 192-347 in the middle of these $\uparrow \uparrow$ strands is only somewhat more stable $\left(\mathrm{t}_{1 / 2} \sim 198 \mathrm{~h}\right)(1)$ than $14-1 \mathrm{~B}$, because it may still allow collapse of RCL and its partial insertion. However replacing flexible glycine G355 (P14) with stiff cysteine trapped in the disulfide bridge (with C197) keeps T356-T357 out, hinders opening of the hydrogen bonds zipper and the incorporation of P13-P4 (T356 $\rightarrow \mathrm{V} 366)$ fragment of RCL as the antiparallel $\downarrow \uparrow \downarrow \uparrow \downarrow \beta$-strand.

The placement of mutations in the discussed structures are far from the RCL hinge region (except for $\mathrm{W} 198 \mathrm{~F}$ in $3 \mathrm{q} 02$ and cysteines in VLHL-PAI-1) and are slowing the movement of the structural subdomains necessary for the active $\rightarrow$ latent transition by affecting local charge and hydrophobicity. The W198F single mutation (3q02) eliminates the hydrogen bond W198 NE1 to N352 O which is observed in some active as well as in latent structures and thus might be irrelevant. The interactions between aromatic moieties of W(or F)198...Y251... F194 are preserved and it must be this aromatic moiety that is crucial in the rate-limiting step of the serpin reaction, i.e. release of $\mathrm{P}^{\prime}$-side of the cleaved RCL from the substrate pocket of the proteinase, a step which highly depends on the structure of the serpin breach region (7). However, from the structural point of view the notable improvement in serpin's stability [18 times better than wt-PAI-1 (18)] seems to be resulting from the combination of the subtle changes in the hydrophobic core caused by this single mutation to an aromatic but smaller size residue. Another pertinent observation is the helical secondary structure of the region where D173H and K177T mutations are in 14-1B mutants (1b3k, 1dvm, 1db2), which otherwise shows distorted coil for the native sequence both in the active (3q02, 3r4l) (Fig. 1) and the latent (1c5g, 1dvn,1lj5) forms of PAI-1.

Sugar moieties in the glycosylated enzymes are notorious for disorder, often not reported in the PDB-deposited structures, and thus not accounted for in modeling such as molecular binding in in silico calculations, for example when seeking potential inhibitors. Among the PDB entries for PAI-1 their partial structure is determined in 1a7c where two glycosylation sites, N232-NAG and N288-NAG-NAG-RIP (NAG, N-acetyl-D-glucosamine; RIP, ribose), are reported. The presence and the structure of the sugars depend on the expression system. The protein used in our studies was expressed in Spodoptera frugiperda (Sf9) and expected to be 'humanized' in terms of its glycan (19). Vertebrates (including human) produce complicated glycans starting with

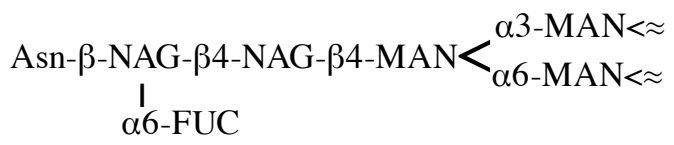

while insects create similar N-glycan structures but much shorter, encompassing only the above fragment with an additional second $\alpha 3-F U C$ at the first NAG (MAN, mannose; FUC, fucose). Our X-ray analysis confirmed glycosylation at N232 and N288 showing: N232, NAG, which clearly extends further but is disordered, and N288, NDG(FUC)- NAG-BMA-BMA which is more orderly but may also be incomplete [Fig. 2b, NDG, 2-(acetylamino)-2-deoxy- $\alpha$-D-glucopyranose; BMA, $\beta$-D-mannose according to the PDB notation].

These two structures (3q02 with W198F and the 3r41 reported here with Q197C, G355C) confirm that even a small alteration in the vicinity of Gly355 hinge can have a very significant role in severe retardation of the serpin's spontaneous 
transition to the latent state. In contrast to the W198F mutant whose transition to the latent form is irreversible, the activity of VLHL-PAI-1 is preserved much longer and can be turned off and on by breaking or reconstituting the disulfide bridge (20). Over 600 PAI-1 variants were constructed to study its structure-function relationship (18), many to extend $t_{1 / 2}$ of this protein and VLHL PAI- 1 has the longest $t_{1 / 2}$. Thus VLHL PAI-1 with its very long, controllable activity and high structural identity with wt-PAI-1 is a good candidate for a personalized, molecular therapy to aid patients with ailments $(21,22)$ that may be positively affected by its utilization.

\section{Acknowledgements}

E.S.J and J.J. wish to thank Dr Richart Hart from PharmaIP for the grant N-121217-01, keen interest, advice and an unfailing support of this research. This work was supported in part by a grant from the Stranahan Endowment Fund for Oncological Research.

\section{References}

1. Chorostowska-Wynimko J, Swiercz R, Skrzypczak-Jankun E, Wojtowicz A, Selman SH and Jankun J: A novel form of the plasminogen activator inhibitor created by cysteine mutations extends its half-life: relevance to cancer and angiogenesis. Mol Cancer Ther 2: 19-28, 2003.

2. Stout TJ, Graham H, Buckley DI and Matthews DJ: Structures of active and latent PAI-1: a possible stabilizing role for chloride ions. Biochemistry 39: 8460-8469, 2000.

3. Tucker HM, Mottonen J, Goldsmith EJ and Gerard RD: Engineering of plasminogen activator inhibitor-1 to reduce the rate of latency transition. Nat Struct Biol 2: 442-445, 1995.

4. Zhou A, Huntington JA, Pannu NS, Carrell RW and Read RJ: How vitronectin binds PAI-1 to modulate fibrinolysis and cell migration. Nat Struct Biol 10: 541-544, 2003.

5. Nar H, Bauer M, Stassen JM, Lang D, Gils A and Declerck PJ: Plasminogen activator inhibitor 1. Structure of the native serpin, comparison to its other conformers and implications for serpin inactivation. J Mol Biol 297: 683-695, 2000.

6. Lin Z, Jiang L, Yuan C, et al: Structural basis for recognition of urokinase-type plasminogen activator by plasminogen activator inhibitor-1. J Biol Chem 286: 7027-7032, 2011.
7. Blouse GE, Perron MJ, Kvassman JO, et al: Mutation of the highly conserved tryptophan in the serpin breach region alters the inhibitory mechanism of plasminogen activator inhibitor-1. Biochemistry 42: 12260-12272, 2003.

8. Jensen JK, Thompson L, Nissen P, et al: Crystal structure of plasminogen activator inhibitor-1 in an active conformation with normal thermodynamic stability. J Biol Chem: Jun 21, 2011 (Epub ahead of print).

9. Xue Y, Bjorquist $\mathrm{P}$, Inghardt $\mathrm{T}$, et al: Interfering with the inhibitory mechanism of serpins: crystal structure of a complex formed between cleaved plasminogen activator inhibitor type 1 and a reactive-centre loop peptide. Structure 6: 627-636, 1998.

10. Jankun J, Aleem AM, Selman SH, Basrur V and SkrzypczakJankun E: VLHL plasminogen activator inhibitor spontaneously reactivates from the latent to active form. Int J Mol Med 23: 57-63, 2009.

11. Lee HJ and Im H: Purification of recombinant plasminogen activator inhibitor-1 in the active conformation by refolding from inclusion bodies. Protein Expr Purif 31: 99-107, 2003.

12. Otwinowski $Z$ and Minor W: Processing of X-ray diffraction data collected in oscillation mode. Methods Enzymol 276: 307-326, 1997.

13. Collaborative Computational Project Number 4, The CCP suite: programs for protein crystallography. Acta Crystallogr D Biol Crystallogr 50: 760-763, 1994.

14. Emsley P and Cowtan K: Coot: model-building tools for molecular graphics. Acta Crystallogr D Biol Crystallogr 60: 2126-2132, 2004.

15. DeLano WL: The PyMOL Molecular Graphics System (2002). DeLano Scientific, San Carlos, CA, USA. Available from, http:// www.pymol.org.

16. DeLano WL: The case for open-source software in drug discovery. Drug Discov Today 10: 213-217, 2005.

17. Krissinel $\mathrm{E}$ and Henrick $\mathrm{K}$ : Multiple alignment of protein structures in three dimensions. CompLife LNBI 3695: 67-78, 2005.

18. De Taeye B, Gils A and Declerck PJ: The story of the serpin plasminogen activator inhibitor 1: is there any need for another mutant? Thromb Haemost 92: 898-924, 2004.

19. Rendić D, Wilson IBH and Paschinger K: The glycosylation capacity of insect cells. Croatica Chemica Acta 81: 7-21, 2008.

20. Jankun J, Aleem AM, Specht Z, et al: PAI-1 induces cell detachment, downregulates nucleophosmin (B23) and fortilin (TCTP) in LnCAP prostate cancer cells. Int J Mol Med 20: 11-20, 2007.

21. Jankun J, Selman SH, Keck RW, Lysiak-Szydlowska W and Skrzypczak-Jankun E: Very long half-life plasminogen activator inhibitor type 1 reduces bleeding in a mouse model. BJU Int 105: 1469-1476.

22. Swiercz R, Keck RW, Skrzypczak-Jankun E, Selman SH and Jankun J: Recombinant PAI-1 inhibits angiogenesis and reduces size of LNCaP prostate cancer xenografts in SCID mice. Oncol Rep 8: 463-470, 2001. 